\title{
VITAMIN D IMPACT ON HUMAN HEALTH AND ITS RELATION WITH SEVERAL DISEASES
}

\author{
MOAYAD AL-SHAHWAN, SABRINA AIT GACEM*, SHAHD SHAMSEDDIN, MARWA SAMMOUR
}

College of Pharmacy and Health Sciences, Ajman University, UAE

Email: sabrinaaitgacem@yahoo.com

Received: 26 Jul 2018, Revised and Accepted: 21 Sep 2018

\begin{abstract}
Vitamins are organic nutrients and substances that are needed for many biochemical functions. They are one of the six essential elements required for human life. Vitamin D is also known among the public as the Sunshine vitamin, as it is produced internally within the human body when exposed
\end{abstract} to sunlight and it is well known that the big majority of United Arab Emirates (UAE) population suffer from its deficiency.

They are usually not found inside the body and therefore must be eaten from food or taken by supplements. Vitamin D that is obtained from diet or body synthesis when it is exposed to sufficient sunlight is biologically inactive without the enzymatic activation that converts it into its active form in the liver and kidney.

Deficiency of vitamin D could lead to many troubling diseases such as depression including seasonal affective disorder, Parkinson's disease, Alzheimer's disease, Arthritis (gout, osteoarthritis, tendonitis), Osteoporosis, gum and teeth diseases, obesity, diabetes, heart diseases, metabolic syndromes, immunity related diseases (sclerosis, erythematosis), and cancer, which will be discussed in this review. The main objective of the current review is to evaluate the associations of vitamin D with the diverse health outcomes according to the latest studies done so far.

Keywords: Vitamin D, Cancer, Bone health, Mental health

(C) 2018 The Authors. Published by Innovare Academic Sciences Pvt Ltd. This is an open access article under the CC BY license (http://creativecommons.org/licenses/by/4.0/) DOI: http://dx.doi.org/10.22159/ijap.2018v10i6.28776

\section{INTRODUCTION}

For over 90 y ever since researcher Elmer McCollum had discovered Vitamin D, and scientists are trying to investigate it, there has been many researches in the field of vitamin D and now it's a well-known vitamin that affect almost all the organs in the body. However, for the general population and even doctors they still don't truly comprehend vitamin D impact, that is why it's important to have a full understanding about it [1].

During sunlight exposure, the skin is penetrated by ultraviolet B photons by which pre-vitamin D3 is isomerized into vitamin D3 by the body heat [2]. Most people depended on the sun for the need for vitamin $\mathrm{D}$ but when they use sunscreen, ant-aging creams they block the effect of the sun and reduce their synthesis, other factors like their exposure time of the day, and the season, and latitude they all dramatically affect the skin pigment and disturbs synthesis of previtamin D3 [3]. It is necessary to update the evaluation of the positive effect of mild sunlight to make sure that all people take their need of vitamin D to gain health [4]. Vitamin D deficiency causes rickets and growth retardation and an increased risk of adult fractures $[5,6]$. It is also associated with other conditions such as increased risk of common cancers, infectious diseases, cardiovascular diseases and other diseases $[7,8]$.

Vitamin D has a vital role in maintaining good health and its benefits or importance comes from its role in absorbing calcium and phosphorus from the gastrointestinal track, that's why it can be used to treat and prevent bones and muscle aches as well as chronic fatigue, teeth and dental problems, and osteoporosis [9]. It also plays a role in controlling the normal and cancerous cells therefore it could be used to prevent or treat cancers of breasts, colon, pancreas, and prostate [8]. It's also well-known in its effect in the immune system and the diseases related to immunity as it can boost it up when there is sufficient intake of vitamin $D$, or it lowers the immunity down when a person having vitamin D deficiency $[5,10]$. Researches also indicate its role in the kidney and the heart as it inhibits the Renin Angiotensin Aldosterone System (RAAS) [6, 9]. Vitamin D also stimulates the production of insulin from the cells in the pancreas and prevent insulin resistance, therefore vitamin D is important for type 2 diabetes mellitus and even type 1 diabetes when it's related to immunity $[6,11]$. The most common health benefit of vitamin $D$ is its contribution in maintaining skin function and preventing diseases related to it such as psoriasis and skin cancer $[8,12]$. Not to forget its rule in mental health as well. Vitamin D affects mood disorders, such as depression, and other mental diseases $[13,14]$. The current review article includes data extracted from databases such as Google scholar and Embase. The titles and abstracts obtained through the electronic searches were identified and checked for their relevance and studies to be included in the review were selected. The current research took place from January 2018 to June 2018 and the selected studies to be included in this review were filtered to include studies from 1999 until 2018. Restrictions on language of publication imposed, as only relevant studies published in English were included. The main objective of the current review is to evaluate the associations of vitamin D with the diverse health outcomes according to the latest studies done so far.

\section{Vitamin $D$ and different malignancies}

Vitamin D is said to play a very important role in controlling many malignant cells and was found to be useful in preventing different cancers and an insufficiency of vitamin D contributes in mutating cells and leading to cancer [8]. It is suggested in some studies that vitamin D may contribute to the protection from cancer and this theory was based on the vitamin D receptor (VDR) sites in several cancer cells and researchers believe that the vitamin can block the growth of malignant cells and promote their transformation into non-malignant cells and some studies claimed that vitamin D can regulate certain cell processes such as cell proliferation in which, 1a,25 (OH)2D3, which is the active metabolite of vitamin D eliminates cell proliferation [15]. It can regulate other processes as well such as differentiation, and inhibition of angiogenesis and some studies confirm the assumption that this vitamin could have an impact in lowering the risk of cancer $[16,17]$.

A new study of 2018 by the International Agency for Research on Cancer, which is the largest observational study to date on the relationship between vitamin $\mathrm{D}$ and cancer, revealed that no relation was found between lung cancer risk and vitamin D levels in the blood. It presents an analysis of more than 5300 case-control pairs within 20 international prospective cohort studies. This large recent study was held to provide an accurate answer as to whether circulating vitamin D may affect lung cancer risk. Instead of relying only on self-reported smoking data like was done in the previous, smaller studies, this study included an analysis of a nicotine 
metabolite found in blood called cotinine, which provided an objective measurement of recent tobacco exposure [17].

A recent large cohort study on United States women held in 2018 on the association between Vitamin D genetic risk score and cancer risk. This study revealed that there was not a relation between them [18]. A study in 2014 found that supplements are recommended for people with cancer and a decreased level of vitamin D can have a bad outcome for some cancers and that higher levels during diagnosis are associated with better results and it was concluded that vitamin D3 could reduce the risk of cancer, but data quality problems were noted in this study [19].

Some studies claim that there is a link between vitamin D that is circulating in the blood and prostate and colorectal cancer [20].

A recent study of 2017 in Finland showed that men with higher levels of vitamin D had a decreased risk of having rectal and colon cancer [9]. Similarly, a $67 \%$ lower risk of colorectal cancer was observed among women as well [20].

Some results of some studies reflected a positive relation between vitamin D levels in the blood serum and its protective effect from prostate cancers [21]. Some environmental studies have found as well a link between increased latitude and the increased burden of prostate cancer. In addition to a study in Spain, an investigation showed only such a relationship for the northern US districts, in the southern counties there was no significant association $[9,22]$.

Some studies found that there was no significant relation between taking supplements of vitamin $\mathrm{D}$ and breast cancer, but due to the small size of the study, this limitation could not enable the authors to detect statistically significant results and correlations [23].

Investigations made on immigrants have shown that melanomas are less common in humans who migrated from a place with low sunlight to a place of high sunlight and a meta-analysis of solar radiation studied the effect of the sunlight and risk of melanoma which occurs on the stem associated with the relatively young age and the emergence of maternal marks and many of them have a mutation in the proto-oncogene. Melanomas occurring in normal sun-protected areas, on the other hand, tend to occur at a later age [12, 24].

\section{Vitamin D and mental health}

Vitamin D is a very important natural fatty organic substance that is needed in many metabolic functions as explained and one of the most organs that needs the product of vitamin $\mathrm{D}$ is the brain. The association of vitamin D deficiency has been linked with many mental disorders, and the supplementation of vitamin D also was found to prevent and treat such diseases [25].

It was observed in a study of 2018 that Vitamin D, as well as some other vitamins, may be responsible factors for schizophrenia, but it is recommended to conduct more extensive studies to observe schizophrenia [26].

Strong evidence confirms that vitamin D plays a huge role in the healthy functioning of the brain [13]. Those with a vitamin D deficiency had a lower scoring on a questionnaire compared with patients with higher levels of vitamin $\mathrm{D}(\mathrm{P}<0.001)$ and the same researchers examined a larger sample of 5,596 women living in communities in France (mean age of $80 \mathrm{y}$ ) and found that those with insufficient dietary intake of vitamin D had significantly lower average cognitive scores [22, 27].

A study showed that in young people, a deficient vitamin D level has a significantly higher level of depression compared to adults who had an effective amount of vitamin D $[13,28]$. A randomized clinical trial in which subjects who have either vitamin $\mathrm{D}$ or placebo showed that the effects of a one year course of vitamin D on the mood were improved to a large extent and participants who received 40,000 IU of vitamin D had greater improvement in depression and mood indices while a lesser improvement was observed in patients with 20,000 IU of vitamin D and without changes with placebo [29, 13, 27-32].

A seasonal affective disorder study, randomized either to phototherapy or to the addition of vitamin $\mathrm{D}$, to check the results of vitamin D therapy in these cases. Participants treated with phototherapy remained the same, while participants treated with vitamin D showed improvement in psychological tests [27, 33]. There is a big risk for some women during pregnancy with excessive intake of vitamin $\mathrm{D}$ to lead to mental retardation in her coming child, as it was reported in a study that women who daily swallowed from 2000 to 3000 units of vitamin D, drinking milk and consuming another rich vitamin D meal ended with a child who was mentally retarded, had heart defects, high blood pressure and some kidney disorders. That's why it's very important to stay within the recommended requirement of vitamin $D[14,27]$.

A study in which more than 1,600 elderly people were observed for 6 y showed that people suffering from severe vitamin D deficiency are more likely to develop dementia and Alzheimer's disease than those who have normal levels. Patients who had only a weak deficit had an increased risk of disease by 53\% [14, 30, 34].

Evidence shows that people with Parkinson's have some abnormalities in the endocrine system of vitamin D. These include low bone mineral density (BMD), a vitamin D deficiency and an increase in bone tissue producers that are urinary $\mathrm{N}$-end telopeptide of type I collagen and bone alkaline phosphatase [14, 34]. Exposure to sunlight can increase the BMD of patients with Parkinson's disease by increasing the levels of 25-hydroxyvitamin D3 (250HD) in the serum, which they usually have a flaw. However, it remains unknown whether vitamin D deficiency is the cause or the consequence of Parkinson's disease, but it certainly plays an important role in it. Vitamin D Receptor and $1 \alpha$-hydroxylase, the enzyme responsible for the formation of active vitamin $D$ in the brain were detected in both large substantia nigra (SN) neurons of the hypothalamus [14,34]. There is evidence of the involvement of vitamin $D$ in the function of the brain of mammals.

\section{Vitamin D and cardiovascular diseases}

Vitamin D deficiency is connected to high myocardial diseases and cardiovascular risk; however, the way it works is yet to be investigated in further details [35]. Studies show the role of vitamin D on the heart as it hinders the Renin-Angiotensin-aldosterone system (RAAS). At the point when RAAS is overactive, it can cause many maladies for example, heart failure, inflammation of blood vessels, hypertension and others. An expansion in vitamin D consumption decreases the danger of chronic heart disease as it was shown in an investigation of African American participants in which vitamin D deficiency was seen in the high majority of patients with heart failure $[6,7]$.

A recent study held in 2018 found a significant association between low plasma vitamin $D$ levels and prevalence of hypertension whereas, no significant association between low plasma vitamin D levels and prevalence of diabetes mellitus was found. There was a statistically significant correlation between vitamin $\mathrm{D}$ deficiency and acute coronary syndrome, even after controlling for traditional cardiovascular risk factors $(\mathrm{P}=0.028)$ and it can be concluded that Vitamin D deficiency is independently associated with acute coronary syndromes, and could be an independent risk factor for the acute coronary syndrome, but clinical trials are urgently needed to determine whether this represents a causal association and whether vitamin $D$ replacement therapy can reduce the associated increase in cardiovascular risk [36].

Several investigations demonstrated a relationship with calcium metabolism issues with an increased risk of hypertension occurrence [6,22].

Some studies propose that increased blood concentrations of vitamin D can decrease the risk of hypertension [37]. Some metaanalyses studies have proposed that vitamin D had an antihypertensive effect. However, other studies could not affirm this effect [38]. A study found that the supplementation of calcium in addition to vitamin $\mathrm{D}$ did not diminish the danger of chronic heart disease or stroke and did not have an effect on the incident of hypertension $[39,40]$.

\section{Vitamin D and diabetes mellitus}

Some investigations have announced that vitamin $\mathrm{D}$ is critical for typical insulin secretion and glucose resistance, which demonstrates 
that the lack of vitamin D causes less insulin production, and this might be related the availability of receptor sites in the beta cells of the pancreas, from which insulin is secreted or it can be related to the control of vitamin D and calcium level.

1,25-hydroxyvitamin $\mathrm{D}$ is a controller of insulin discharge by pancreatic $\beta$ cells and subsequently, low 25 -hydroxy vitamin D levels are associated with impaired $\beta$-cells [41].

In a recent study of 2018 it was concluded that co-administration of Vitamins D, B9, and B12 exerted some antiatherosclerotic effects through inhibiting lipid peroxidation coupled with increasing aortic tissue nitric oxide (NO). These findings may have important uses and implications in the modulation and protection against diabetic endothelial dysfunction [42].

A study have proposed that Type 2 diabetes can be prevented with adequate intake of vitamin D and this study involved male participants who were asked to ingest a solution with high sugar content and then blood and urine analysis was performed and it was revealed that participants who had a deficiency had some issues with the glucose transfer $[4,11]$. Several studies were done since many years exhibited that VDD in animals suppresses pancreatic insulin secretion showing that vitamin $D$ is fundamental for the pancreas [43]. In contrast, another study showed that in healthy non-diabetic grown-ups treatment with vitamin D had no significant impact on the glucose level [44].

The different results of several studies are mostly due to the small sample size of respondents and more studies are needed to investigate this correlation deeply. Numerous studies have shown an increased prevalence of vitamin D lack in children with type 1 diabetes mellitus, which proposes a relation among the two issues [45].

A study reflecting the biggest assessment of the relationship between blood levels of vitamin $\mathrm{D}$ and diabetes concluded that vitamin D could enhance glycemic control [40]. Some studies have shown that this vitamin can prevent the decrease of nerve factor that is needed for sensory neurons development [46].

\section{Vitamin D and bone health}

It is well seen from the literature that vitamin $D$ has a great impact in the avoidance of many chronic bone diseases. A study that involved participants with bone disorders showed a positive relation between vitamin D deficiency and osteomalacia and rickets $[9,47]$.

In a recent study of 2018 it was concluded that vitamin D supplements only increase bone density in adults with 25hydroxyvitamin $\mathrm{D} \leq 30 \mathrm{nmol} / \mathrm{l}$. This moves us further towards a trialbased definition of vitamin $\mathrm{D}$ deficiency in adults with adequate calcium intakes and suggests that supplement use should be targeted accordingly. Future trials of vitamin D supplementation should focus on individuals with 25-hydroxyvitamin D concentrations in this range [48].

Stress fractures are frequent among youthful competitors and athletes. A study proposed that a low blood level of inactive vitamin D might be a predisposing factor for bone stress fractures [49].

Osteoarthritis is a disease of the synovial membrane, subchondral bone and cartilage, and the low intake and low levels of vitamin $D$ in the blood can exacerbate osteoarthritis and cause the disease to develop [50]. In a study, the results showed that the risk of osteoarthritis had increased three times in participants in the lower and middle groups of vitamin D levels in the blood and it was concluded that low levels of vitamin $\mathrm{D}$ in the blood increase the risk of bone arthritis [51,52].

\section{Vitamin $D$ and other diseases}

\section{Inflammatory and autoimmune diseases}

Vitamin D and a good diet help the immune system to develop clear memories of contagious substances and vaccines for effective protection [10]. Recent investigations showed that vitamin D is critical for the proper production of antimicrobial proteins from immune cells in response to infection, these proteins kill the bacteria. In the absence of these proteins, antibiotics are less effective, and risk of resistance, recurrent infections or developing chronic infections increase [10, 53, 54]. Ultraviolet $B$ (UVB) is important for the synthesis of vitamin D, but it can also inactivate viruses. Low levels of UVB in winter and a drop in vitamin D levels allow more viruses to attack the body and make it vulnerable. Influenza is a perfect example as the high season of flu follows the summer solstice for six months and its global alternates between the northern and southern hemispheres according to seasonal changes in the hemisphere $[55,56]$.

In multiple sclerosis, the immune system produces antibodies against the proteins found in the nerve lining knows as myelin sheath. Damage to the lining leads to a rupture of the neural cover, which must be intact to accelerate the transmission of electrical impulses. Destruction of the coating significantly slows down the transfer of electrical impulses, which leads to a partial or permanent loss of sensation or muscle function. In a study more cases of MS were seen in places far from the equator, except in those places where people consume large amounts of cold water fish, which is rich in vitamin D and omega-3, e. g. Icelandic [57].

In addition, studies on the addition of vitamin D in people with MS showed a decrease in the activity of the disease when they began receiving vitamin D supplements $[13,58]$. Psoriasis is a skin disease that occur almost anywhere on the skin of scalp, elbows, knees, feet, and hindquarters. Many people with psoriasis have an abnormal growth of nails, and increased inflammation of the eyes, joints, and intestine. Modern theories suggest that a low tolerance of bacteria (usually streptococci) on the skin, in the mouth or in the nose, causes an immune response leading to psoriasis $[5,22,13]$.

Psoriasis is known to worsen in the winter and improves in the summer. This seasonal change was studied and found that the activity of the disease is associated with exposure to ultraviolet radiation and vitamin $\mathrm{D}$. In general practice, doctors traditionally treat patients with psoriasis by subjecting them to ultraviolet radiation and topical steroids. For decades, researchers have discovered that activated analogues of vitamin D and vitamin D used as ointments can reduce or eliminate psoriasis and increase the effectiveness of ultraviolet light therapy. A recent study of 2018 observed no association between gingival inflammation and $25(\mathrm{OH})$ D status among the postmenopausal women [59].

\section{Asthma and chronic kidney diseases}

The vitamin D effects the calcium, phosphorus, and parathyroid hormone (PTH) which are important factors for the kidney. Any imbalance or change of the hemostasis of these factors was proven to affect the kidneys badly. The function of kidneys in the production of the active form from the inactive form of this vitamin has been shown, and renal disease has been known to cause VDD and some studies also proposed that VDD has an impact in the progression of kidney disease [60].

A recent study observed many promising preventive strategies emerged from the available results since vitamin D relatively improve pulmonary function in asthmatic patients concomitantly with reduction of inflammatory burden in those patients, suggesting that vitamin D plays effective role to be a good candidate as an adjuvant therapy, but further evaluation is needed to explore the potential effect of vitamin D supplementation in acute or chronic asthma [61].

\section{CONCLUSION}

Vitamin D is an important vitamin that impacts many systems in the body and the deficiency in its levels lead to severe issues that could have been avoided if proper levels were maintained.

It is very important to correct the vitamin levels as it plays major roles in the body function, and keeping vitamin D balanced is a very important factor in the treatment of much variety of diseases and preventing a lot more of them. Neither too much vitamin D nor too little is good for the human health so in order to maintain the human body in its optimum mental and physical health, a balanced state in the vitamin level should be acquired. It can be concluded that although many controversies still exist suggesting that vitamin $D$ is 
not connected to any health condition and it is just a marker of general good health, but there is also good evidence of the relation between vitamin D level and malignancies as well as mental health, bone health and other health issues. Therefore, to clarify the exact role of vitamin D well-designed high-quality studies should be conducted and further studies are required to study the efficacy of vitamin D supplementation in reducing cancer risk.

\section{AUTHORS CONTRIBUTIONS}

All the author have contributed equally

\section{CONFLICT OF INTERESTS}

\section{Declared none}

\section{REFERENCES}

1. Deluca HF. History of the discovery of vitamin D and its active metabolites. Bonekey Rep 2014;3:1-8.

2. Wacker M, Holick MF. Sunlight and vitamin D. Dermatoendocrinol 2013;5:51-108.

3. Hume EM, Lucas NS, Smith HH. On the absorption of vitamin d from the skin. Biochem J 1927;21:362-7.

4. Hossein A, Holick MF. Vitamin D for health: a global perspective. Mayo Clin Proc 2013;88:720-55.

5. Zaidi S. Power of vitamin d. Endocr Pract 2012;16:3-99.

6. Anderson JL, May HT, Horne BD, Bair TL, Hall NL, Carlquist JF, et al. Relation of vitamin D deficiency to cardiovascular risk factors, disease status, and incident events in a general healthcare population. Am J Cardiol 2010;106:963-8.

7. Wang L, Song Y, Manson JE, Pilz S, März W, Michaëlsson K, et al. Circulating levels of 25hydroxy-vitamin $d$ and risk of cardiovascular disease: a meta-analysis of prospective studies. Circ Cardiovasc Qual Outcomes 2012;5:819-29.

8. Barry M, Giovannucci E, Devries E, Møller H. Vitamin D and cancer. Int Agency Res Cancer 2008;5:4-303.

9. Tiwari P, Sharma N. Role of vitamin d in various illnesses. J Pharm Care Heal Syst 2017;4:3.

10. Beard JA, Bearden A, Striker R. Vitamin D and the anti-viral state. J Clin Virol 2011;50:194-200.

11. Nakashima A, Yokoyama K, Yokoo T, Urashima M. Role of vitamin $\mathrm{D}$ in diabetes mellitus and chronic kidney disease. World J Diabetes 2016;7:89.

12. Vieth R. Effects of vitamin $d$ on bone and natural selection of skin color: how much vitamin D nutrition are we talking about. Bone Loss Osteoporos 2003;9:135-50.

13. Thacher TD, Clarke BL. Vitamin D insufficiency. Mayo Clin Proc 2011;86:50-60.

14. Balion C, Griffith LE, Strifler L, Henderson M, Patterson C, Heckman G, et al. Vitamin D cognition and dementia; a systematic review and meta-analysis. Neurology 2012;79:1397-405.

15. Thorne J, Campbell MJ. The vitamin D receptor in cancer. Proc Nutr Soc 2008;67:115-27.

16. Byers T. Editorial anticancer vitamins du jour-the ABCED's so far. Am J Eipemiol 2010;172:5-7.

17. Muller DC, Hodge AM, Fanidi A, Albanes D, Mai XM, Shu XO, et al. No association between circulating concentrations of vitamin D and risk of lung cancer: an analysis in 20 prospective studies in the lung cancer cohort consortium (LC3). Ann Oncol 2018;29:1468-75.

18. Chandler P, Tobias D, Wang L, Smith S, Chasman D, Rose L, et al. Association between vitamin $\mathrm{D}$ genetic risk score and cancer risk in a large cohort of U. S. women. Nutrients 2018;10:55.

19. Buttigliero C, Monagheddu C, Petroni P, Saini A, Dogliotti L, Ciccone G, et al. Prognostic role of vitamin d status and efficacy of vitamin d. Suppl Cancer Patients 2011;16:1215-27.

20. Feskanich D, Ma J, Fuchs CS. Plasma vitamin D metabolites and risk of colorectal cancer in women. Cancer Epidemiol Biomarkers Prev 2004;13:1502-8.

21. Freedman DM, Chang SC, Falk RT. Serum levels of vitamin D metabolites and breast cancer risk in the prostate, lung, colorectal, and ovarian cancer screening trial. Cancer Epidemiol Biomarkers Prev 2008;17:889-94.

22. Holick MF. High prevalence of vitamin D inadequacy and implications for health. Mayo Clin Proc 2006;81:353-73.
23. Mithal A, Bansal B, Kyer CS, Ebeling P. The asia-pacific regional audit-epidemiology, costs, and burden of osteoporosis in India: a report of international osteoporosis foundation. Indian J Endocrinol Metab 2014;18:449-54.

24. Egan KM. Vitamin D and melanoma. Ann Epidemiol 2009;19:455-61.

25. Vieth R. Vitamin D supplementation, 25-hydroxyvitamin D concentrations. Am Soc Clin Nutr J 1999;25:842-56.

26. Jose R, Venketeswaramurthy N, Sambath KR. A critical review on the hypothesis, pathophysiology of schizophrenia, and role of vitamins in its management. Asian J Pharm Clin Res 2018;11:25.

27. Graham KA, Keefe RS, Lieberman JA, Calikoglu AS, Lansing KM, Perkins DO. Relationship of low vitamin D status with positive, negative and cognitive symptom domains in people with firstepisode schizophrenia. Early Int Psychiatry 2015;9:397-405.

28. Eskandari F, Martinez P, Torvik S. Low bone mass in premenopausal women with depression. Arch Intern Med 2007;167:2329-36.

29. Bikle DD. Vitamin D metabolism, mechanism of action, and clinical applications. Chem Biol 2014;21:319-29.

30. Ganji V, Milone C, Cody MM, Mccarty F, Wang YT. Serum vitamin D concentrations are related to depression in young adult US population: the third national health and nutrition examination survey. Int Arch Med 2010;3:29.

31. Jorde R, Sneve M, Figenschau Y, Svartberg J, Waterloo K. Effects of vitamin D supplementation on symptoms of depression in overweight and obese subjects: randomized, double-blind trial. J Intern Med 2008;264:599-609.

32. Olmos A, Avila E, Durand M, Diaz L. Regulation of calcitriol biosynthesis and activity: focus on gestational vitamin D deficiency and adverse pregnancy outcomes. Nutrients 2015;7:443-80.

33. Gloth F, Alam WB. Vitamin D vs broad spectrum phototherapy in the treatment of the seasonal affective disorder. J Nutr Heal Aging 1999;3:5-7.

34. Evatt M, Delong M. Prevalence of vitamin d insufficiency in patients with parkinson disease and Alzheimer disease. Arch Neurol 2008;65:1348-52.

35. Grober U, Spitz J, Reichrath J, Kisters K, Holick MF. Vitamin D: rickets prophylaxis to general preventive health care. Dermatoendocrinol 2013;5:331-47.

36. Hallak A, Malhis M, Abajy M. Vitamin-d deficiency and risk of acute coronary syndrome. Int J Pharm Pharm Sci 2018;10:171.

37. Beveridge LA, Struthers AD, Khan F, Jorde R, Scragg R. Effect of vitamin $D$ supplementation on blood pressure: a systematic review and meta-analysis incorporating individual patient data. JAMA Intern Med 2015;175:745-54.

38. Aldujaili EAS, Munir N, Iniesta RR. Effect of vitamin D supplementation on cardiovascular risk factors and exercise performed in healthy participants: a randomized placebocontrolled preliminary study. Ther Adv Endocrinol Metab 2016;7:153-65.

39. Hsia J, Heiss G, Ren H. Calcium and vitamin D supplementation and cardiovascular events. Circulation 2007;115:846-54.

40. Margolis KL, Ray RM, VanHorn L. Effect of calcium and vitamin D supplementation on blood pressure: the women's health initiative randomized trial. Hypertension 2008;52:847-55.

41. Peterlik M, Cross HS. Vitamin D and calcium deficits predispose for multiple chronic diseases. Eur J Clin Invest 2005;35:290-304.

42. Aljoufi F, Elbana M, Tewfik I, Anwar M. Efficacy of cosupplementation therapy with vitamins $\mathrm{b} 9, \mathrm{~b} 12$, and $\mathrm{d}$ on endothelial dysfunction in streptozotocin-induced diabetic rats. Asian J Pharm Clin Res 2018;11:407-10.

43. Takiishi T, Gysemans C, Bouillon R, Mathieu C. Vitamin D and diabetes. Rheumatic Diseases Clinics of North America 2012;38:179-206.

44. Tai K, Need AG, Horowitz M, Chapman IM. Glucose tolerance and vitamin D: effects of treating vitamin D deficiency. Nutrition 2008;24:950-6.

45. Mathieu C, Gysemans C, Giulietti A, Bouillon R. Vitamin D and diabetes. Diabetologia 2005;48:1247-57.

46. Hisatake J, O'kelly J, Uskokovic MR. Novel vitamin D analog, 21(3-methyl-3 hydroxy-butyl)-19-nor D, that modulates cell 
growth, differentiation, apoptosis, cell cycle, and induction of PTEN in leukemic cells. Blood 2001;97:2427-33.

47. Ritu G, Gupta A. Vitamin D deficiency in India: prevalence, causalities and interventions. Nutrients 2014;6:729-75.

48. Macdonald H, Reid I, Gamble G, Fraser W, Tang J, Wood A. 25Hydroxyvitamin D threshold for the effects of vitamin D supplements on bone density: secondary analysis of a randomized controlled trial. J Bone Mineral Res 2018;33:1464-9.

49. Välimäki VV, Alhan H, Lehmuskallio E. Risk factors for clinical stress fractures in male military recruits: a prospective cohort study. Bone 2005;37:267-73.

50. Samuels J, Krasnokutsky S, Abramson B. Osteoarthritis: a tale of three tissues. Bull NYU Hospital Joint Diseases 2008;66:244-50.

51. Garfinkel RJ, Dilisio MF, Agrawal DK. Vitamin D and its effects on articular cartilage and osteoarthritis. Orthop J Sports Med 2017;5:1-8.

52. Mabey T, Honsawek S. Role of vitamin D in osteoarthritis: molecular, cellular, and clinical perspectives. Int J Endocrinol 2015;6:1-14.

53. Pinto R, Pietropaoli D, Ferri C, Cominelli F. Association between inflammatory bowel disease and vitamin d deficiency: a systematic review and meta-analysis. Inflamm Bowel Dis 2016;21:2708-17.
54. Harvey NC, Cantorna MT. Vitamin D and the immune system. J Investig Med 2013;59:244-63.

55. Kearns MD, Alvarez JA, Seidel N, Tangpricha V, Affairs V. The impact of vitamin D on infectious disease. Am J Med Sci 2016;349:245-62.

56. Bergman P, Lindh ÅU, Björkhem L, Lindh JD. Vitamin D and respiratory tract infections: a systematic review and metaanalysis of randomized controlled trials. PLoS One 2013;8:6.

57. Yetley E, Danielle BM, Cheney C, Davis K, Esslinger P, Fischer W. Dietary reference values for vitamin D. EFSA J 2016; 14:4547.

58. Sintzel MB, Rametta M, Reder AT. Vitamin D and multiple sclerosis: a comprehensive review. Neurol Ther 2017;27:59-85.

59. Fernando R, Masulili S, Lessang R, Kusdhany I. Association between the degree of gingival inflammation and 25-hydroxy vitamin d status in postmenopausal women. Int J Appl Pharm 2018;9:95.

60. Zhang Z, Sun L, Wang Y. Renoprotective role of the vitamin D receptor in diabetic nephropathy. Kidney Int 2008;73:163-71.

61. Abbas RS, Abdulridha MK, Shafek MA. Clinical evaluation of the potential anti-inflammatory effect of vitamin D3 adjuvant therapy for chronic asthma in Iraqi patients. Int J Pharm Pharm Sci 2017;9:139-44. 